permutations obtained are no longer random. Consider the algorithm where at the $k$ th step, the $k$ th object is exchanged with any one of the $N$ objects. This transformation when applied $N$ times produces $N^{N}$ equiprobable mappings of a particular permutation into the set of all permutations of $N$ objects. But there are only $N$ ! members in the set and $N^{N}$ is not divisible by $N$ ! if $N>2$, so that each of the $N$ ! permutations is not equiprobable.

The pairwise exchange method is equally well suited for the generation of permutations, combinations, or arrangements. A random combination or arrangement of $p$ objects among $N$ is obtained by performing only $p$ exchanges, the result being the $p$ first objects so generated.

Computing Center

California Institute of Technology

Pasadena, California 91109

1. B. Jansson, Random Number Generators, V. Pettersons Bokindustri Aktiebolag, Stockholm, 1966, pp. 189-191.

2. D. H. Lehmer, "The machine tool of combinatorics," Applied Combinatorial Mathematics, edited by E. Beckenbach, Wiley, New York, 1964, pp. 19-23. MR 30 \#4687.

\title{
On Finding the Disc of Minimum Radius Containing a Given Set of Points*
}

\author{
By L. J. Bass and S. R. Schubert
}

Recently many combinatorial problems have been found to be amenable to computer solution. This report presents a description of one such problem and its solution.

Let $E$ be a given set of points of finite cardinality in the plane $R^{2}$. The problem is to determine the disc $D_{\min }$ of minimum radius such that $E \subset D_{\min }$.

This is a nontrivial problem. The centroid of $E$ gives no information at all. A complete analytic solution appears extremely difficult, since probably new concepts are required. Moreover, any essentially exhaustive procedure formulated for machine solution is only feasible when $E$ has very small cardinality. However, consideration of a few simple geometrical theorems quickly places the problem in a much more tractable setting.

Definition. Let $E \subset \mathbb{R}^{2}$ be given as above. The convex hull of the extreme points of $E$ is that convex polygon $P$ such that $E \subset P$ and the vertices of $P$ are points of $E$. These vertices are called the extreme points of $E$.

Theorem 1. The extreme points of $E$ completely determine $D_{\min }$.

Proof. This is clear since $E \subset P \subset D_{\min }$.

TheOREM 2. There are at least two extreme points of $E$ which are on the boundary of $D_{\min }$. Moreover, if there are exactly two points, then these are, in fact, the endpoints

* Contract No. AFO4(695)-669. 
of the diameter of $D_{\mathrm{rnin}}$ which coincides with the point-set diameter of $E$.

Proof. The first statement of the theorem is clear. Let $P_{1}, P_{2}$ be the extreme points on the boundary of $D_{\min }, P_{1} P_{2}$ the associated line segment, and suppose that $P_{1} P_{2}$ is not the diameter of $D_{\min }$. Now consider the pencil of circles through $P_{1}$ and $P_{2}$. There must exist in this pencil a circle of maximum radius which contains a point of $E$, say $P_{3}$, different from $P_{1}$ and $P_{2}$. Obviously the circle determined by $P_{1}, P_{2}, P_{3}$ contains $E$ and has a radius strictly less than the radius of $D_{\min }$. Hence, $P_{1} P_{2}$ is the diameter of $D_{\min }$ and clearly must coincide with the point-set diameter of $E$.

Theorem 3. Let $\alpha$ be the point-set diameter of $E$. Then the radius $R$ of $D_{\min }$ is bounded below by $\alpha / 2$ and bounded above by $\alpha / \sqrt{ } 3$.

Proof. Clearly $R$ is bounded from below by $\alpha / 2$. Now consider a circle $C$ with an inscribed equilateral triangle $T$ determined by the three points $a, b, c$ and with side $\alpha . \alpha$ is also the point-set diameter of the set $\{a, b, c\}$. The radius $r$ of $C$ is $\alpha / \sqrt{ } 3$. Now let $T^{\prime}$ be another triangle inscribed in $C$, determined by $a^{\prime}, b^{\prime}, c^{\prime}$ and not equilateral; let $\alpha^{\prime}$ be the point-set diameter of $\left\{a^{\prime}, b^{\prime}, c^{\prime}\right\}$. Then $r=\alpha / \sqrt{ } 3<\alpha^{\prime} / \sqrt{ } 3$.

The computer algorithm for finding $D_{\min }$ is based on determining the set of extreme points of $E$. This is done in the following manner. An initial set of extreme points is selected on the basis of extreme values of the coordinates, that is, the two points with the largest and smallest $x$-coordinates and the two points with the largest and smallest $y$-coordinates. This set consists of two, three, or four points. The general situation is pictured in Fig. 1. $P_{1}, P_{2}, P_{3}, P_{4}$ are the points of $E$ with extreme values of the coordinates, and the polygon determined by these points is the first approximation to the convex hull of $E$. The set $E$ is contained in the rectangle $a, b, c, d$. The designated quadrants contain the points of $E$ eligible for refining the convex hull around the reference segments $P_{1} P_{2}, P_{2} P_{3}, P_{3} P_{4}, P_{4} P_{1}$.

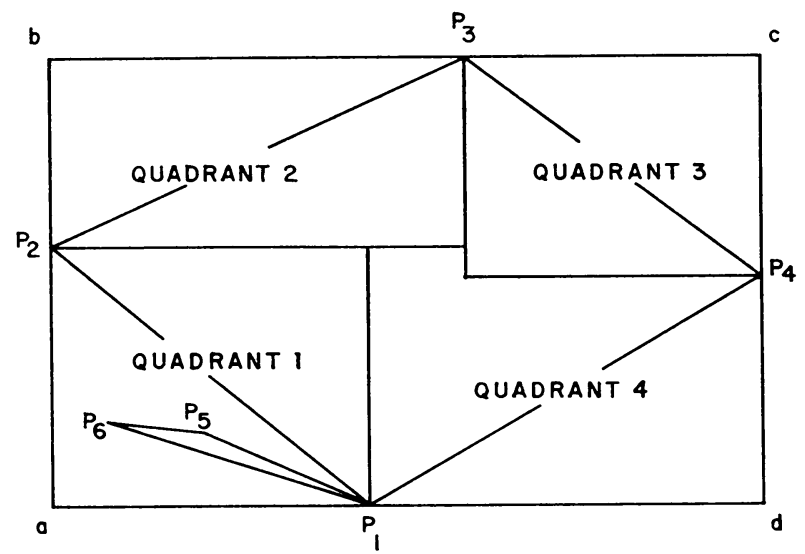

Figure 1. First Approximation to the Hull with a False Extreme Point.

For any line segment $P_{i} P_{j}$, define $s\left(P_{i} P_{j}\right)$ to be its slope. The procedure begins by constructing the portion of the convex hull in the quadrant with diagonal $P_{1} P_{2}$. This is done by ordering the points according to increasing values of the $y$-coordinates and initially comparing the slopes $s\left(P_{1} P_{i}\right)$ with the reference slope $s\left(P_{1} P_{2}\right), i=5,6,7, \cdots$. If $s\left(P_{1} P_{i}\right) \geqq s\left(P_{1} P_{2}\right), P_{i}$ is a candidate for an extreme 
point; if $s\left(P_{1} P_{i}\right)<s\left(P_{1} P_{2}\right), P_{i}$ is never considered again throughout the entire process. As soon as a candidate $P_{i}$ is found, the reference slope is changed to $s\left(P_{i} P_{2}\right)$; thus if, for $j>i, s\left(P_{i} P_{2}\right) \leqq s\left(P_{i} P_{j}\right), P_{j}$ is an extreme-point candidate. Now, if $s\left(P_{1} P_{i}\right)<s\left(P_{i} P_{j}\right), P_{i}$ is a false extreme point since the slopes of the edges of the convex hull must be monotonically nonincreasing as one proceeds clockwise around the hull. The new reference slope is then $P_{j} P_{2}$. For example, as pictured in Fig. $1, P_{5}$ is a fake extreme point that would be discarded. This process proceeds through all four quadrants.

Once the set of extreme points has been found, the point-set diameter $\alpha$ of $E$ is determined among all pairs of extreme points. If the disc with diameter corresponding to the point-set diameter contains $E$, the procedure terminates; if not, the discs corresponding to all triples of extreme points are examined. If the radius of a disc exceeds the bounds specified in Theorem 3, it is discarded. Among the remainder, $D_{\min }$ is determined by considering the discs in the order of increasing radius and seeing whether or not they contain $E$.

The type of geometric approach described in this note was selected over various other approaches specifically because the particular application for which it was used required knowledge of the convex hull of $E$ as well as $D_{\min }$.

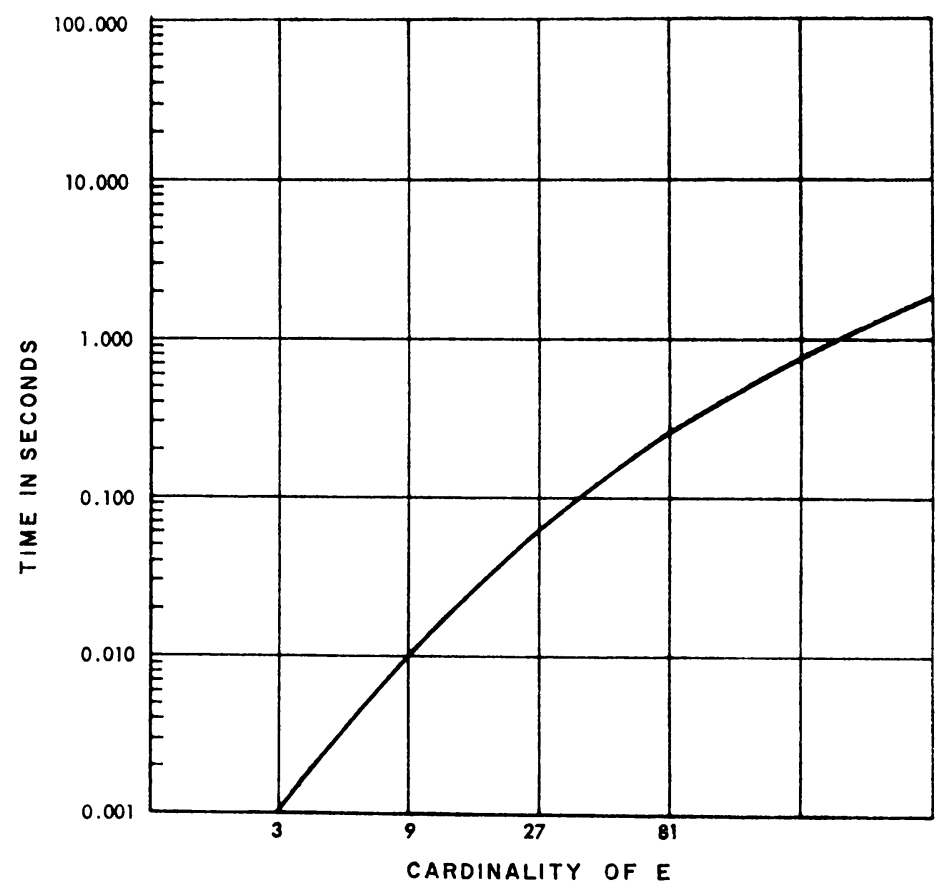

Figure 2. Results of Timing Studies.

The algorithm was programmed for the IBM 7040-7094II Direct Coupled System. The results of a series of timing studies are displayed in Figure 2. 